\title{
CARD15/NOD2 polymorphisms are associated with severe pulmonary sarcoidosis
}

\author{
H. Sato*,\#\#, H.R.T. Williams*,\#,\#\#, P. Spagnolo*, ${ }^{\star}$, A. Abdallah*, T. Ahmad ${ }^{+}$, \\ T.R. Orchard ${ }^{\#}$, S.J. Copley ${ }^{\varsigma}$, S.R. Desai ${ }^{\dagger}$, A.U. Wells*, R.M. du Bois**** and K.I. Welsh*
}

ABSTRACT: Sarcoidosis and Crohn's disease are heterogeneous systemic diseases characterised by granulomatous inflammation. Caspase recruitment domain (CARD)15 is a major susceptibility gene for Crohn's disease, and specifically for ileal and fibrostenotic subtypes. The C-C chemokine receptor (CCR)5 gene has been associated with both parenchymal pulmonary sarcoidosis and perianal Crohn's disease.

This study explored associations between CARD15 polymorphisms, CCR5 haplotype and distinct pulmonary sarcoidosis subtypes. 185 Caucasian sarcoidosis patients were genotyped for CARD15 and CCR5 polymorphisms. The genetic data were compared with 347 healthy controls and were examined for associations with serial pulmonary function tests and chest radiographs. CARD15 genotypes did not differ between the unselected sarcoidosis cohort and controls. However, patients carrying the functional $2104 \mathrm{~T}$ (702W) polymorphism were more likely to have radiographic stage IV disease at 4-yr follow-up. All patients possessing both CARD15 2104T and CCR5 HHC haplotype had stage IV disease at presentation. Carriage of 2104T was associated with worse forced expiratory volume in $1 \mathrm{~s}$, whereas carriage of the CARD15 1761G (587R) polymorphism was associated with better lung function.

For the first time, an association between two CARD15 polymorphisms and specific sarcoidosis phenotypes has been demonstrated, as well as an additive effect of possessing CARD15 2104T and CCR5 HHC haplotype.

KEYWORDS: Caspase recruitment domain 15, C-C chemokine receptor 5, lung function tests, polymorphism, sarcoidosis

arcoidosis and Crohn's disease are idiopathic systemic granulomatous disorders of the lung and intestine, respectively, with variable involvement of the skin, eyes and joints. There are associations between phenotypic variability and human leukocyte antigen class II alleles in both sarcoidosis $\left(D Q B 1^{*} 0201\right.$ with Lofgren's syndrome and erythema nodosum [1]; $D Q B 1^{*} 0602$ with severe pulmonary disease $\left.[1,2]\right)$ and Crohn's disease $\left(D R B 1^{*} 07\right.$ with ileal involvement [3]; DRB1*0103 with extraintestinal manifestations [4]). Furthermore, the HHC haplotype of the $\mathrm{C}-\mathrm{C}$ chemokine receptor (CCR) 5 gene (which promotes $\mathrm{T}$-cell recruitment and activation) is a marker for parenchymal involvement [5] but not for sarcoidosis overall, whereas the $32 \mathrm{bp}$ deletion $(\Delta 32)$ polymorphism of CCR5 is associated with the subgroup of Crohn's patients with perianal disease [6].

The caspase recruitment domain (CARD)15 gene (also known as nucleotide oligomerisation domain $(N O D) 2)$ is a major susceptibility gene for Crohn's disease [7, 8]. The CARD15 gene encodes an intracellular protein (CARD15) of the NOD family, involved in innate immunity through recognition of bacterial pathogen-associated molecular patterns. CARD15 is composed of a nucleotide binding domain and 10 leucinerich repeats $[7,9]$, which recognise muramyl dipeptide, a component of bacterial cell wall peptidoglycan. The three major Crohn's diseaseassociated variants, all located in this region, result in either amino acid substitutions (C2104T $(R 702 W)$ and G2722C (G908R)) or premature truncation of the protein (3020 insertion C $(1007 f s))$. In European and North American cohorts, the risk of disease increases up to threefold with carriage of a single variant allele and 20fold with possession of two variant alleles [10]. Nine other CARD15 single nucleotide polymorphisms (SNPs), including T1761G (R587R) and C802T (P268S), are also associated with

\section{AFFILIATIONS}

${ }^{*}$ Clinical Genomics Group, Royal Brompton Hospital and NHLI, Imperial College London,

\#Dept of Gastroenterology, Imperial College London,

${ }^{\S}$ Radiology Dept, Hammersmith Hospital,

${ }^{f}$ Radiology Dept, King's College

Hospital, London,

+Dept of Gastroenterology, Peninsula Medical School, Exeter, UK,

"Centre for Rare Lung Diseases, University of Modena \& Reggio

Emilia, Modena, Italy.

${ }^{\star}$ National Jewish Health, Denver, CO, USA.

\#\#Both authors contributed equally.

CORRESPONDENCE

H. Sato

Clinical Genomics Group

Royal Brompton Hospital and NHLI Imperial College

1B Manresa Road

London

SW3 6LR

UK

E-mail: h.sato@imperial.ac.uk

Received:

Jan 212009

Accepted after revision:

July 312009

First published online:

Aug 132009 
Crohn's disease, but in these cohorts are in linkage disequilibrium with the three previously mentioned variants [11]. Notably, CARD15 is associated with specific Crohn's subtypes: ileal and fibrostenotic disease.

CARD15 is the disease gene for Blau syndrome, a rare autosomal dominant granulomatous disorder characterised by arthritis, uveitis and a skin rash [12, 13]; early-onset sarcoidosis has also been associated with CARD15 mutations [14]. However, in these diseases, the mutations are distinct from those of Crohn's disease.

The similarities between Crohn's disease and sarcoidosis, and the genotype-phenotype associations observed in the subtypes of the diseases which highlight their heterogeneity, justify the investigation of CARD15 in sarcoidosis. Previous studies of CARD15 in unselected sarcoidosis cohorts have provided conflicting results, with no association found in three studies [15-17], but an association with the G2722C (G908R) polymorphism in one report [18]. However, specific subgroups of sarcoidosis patients were not investigated in any of these cohorts.

Both CARD15 and CCR5 genes are of importance in innate immunity, and have been associated with specific subtypes of Crohn's disease and sarcoidosis. Therefore, carefully phenotyped patients with sarcoidosis were genotyped for 1) five Crohn's disease-associated CARD15 polymorphisms, and 2) the CCR5 HHC haplotype, to test the hypothesis that polymorphisms in these genes would be associated with specific sarcoidosis phenotypes, and to investigate potential gene-gene interactions.

\section{METHODS}

\section{Sarcoidosis patients}

A total of 185 Caucasian patients were included in this study. All were recruited from the sarcoidosis clinic of the Royal Brompton Hospital (London, UK) a tertiary referral centre with patients mainly from the south east of England. In all patients, sarcoidosis was diagnosed histologically, and according to the criteria defined in the American Thoracic Society (ATS)/ European Respiratory Society (ERS)/World Association of Sarcoidosis and Other Granulomatous Disorders (WASOG) consensus statement on sarcoidosis [19].

Written patient consent was obtained from all subjects. The Ethics Committees of the Royal Brompton Hospital gave authorisation for the study.

\section{Controls}

The control population comprised 347 white Caucasian subjects from south-east England, healthy as judged by checks, including medical history, physical examination and routine laboratory blood testing at regular intervals, during a 10-yr period before blood was taken for DNA extraction.

\section{Genotyping for CARD15 and CCR5 polymorphisms}

SNPs were determined using sequence-specific primers and PCR $[20,21]$. Five CARD15 SNPs were studied in all 185 patients (nomenclature according to [11]): $802 \mathrm{C}>\mathrm{T}$ (P268S, exon4, rs2066842), $1761 T>G(R 587 R$, exon 4, rs1861759), 2104C $>T$ (R702W, exon 4, rs2066844), 2722G>C (G908R, exon8, rs2066845) and 3020insC (1007fs, exon 11, rs2066847). Genotyping for eight polymorphisms of the CCR5 gene $(-5563(A / G),-3900(C / A)$, $-3458(T / G),-2459(G / A),-2135(T / C),-2086(A / G),-1835(C / T)$ and 132) was also undertaken as previously described and haplotypes assigned, to identify those possessing the HHC haplotype (-2459G/-2135T/-2086G/-1835C) [5]. 104 of the subjects had been included in the previous study [5] and 56 additional cases were genotyped.

\section{Radiography}

Chest radiographs for each patient were examined and compared in order to determine disease severity and course; these were evaluated independently by two experienced pulmonary radiologists [5] at presentation and 2- and 4-yr follow-up. Chest radiographic data were available for 177 of the 185 patients at presentation to the Royal Brompton Hospital, 158 at 2 yrs and 126 at 4 yrs. These differences were due to the inclusion of a minority of patients with more recent diagnoses who have not been followed up for long enough to be included in the 2- and 4-yr analysis. Chest radiograph staging was classified according to the joint ATS/ERS/ WASOG consensus statement on sarcoidosis [19]. Table 1 shows the chest radiograph staging for patients genotyped for CARD15 polymorphisms only, and for patients genotyped for both CARD15 and CCR5 polymorphisms, respectively.

\section{Pulmonary function testing}

Pulmonary function tests included forced expiratory volume in $1 \mathrm{~s}$ (FEV1) and forced expiratory vital capacity (FVC) assessed by spirometry and transfer factor of the lung for carbon monoxide $(T \mathrm{~L}, \mathrm{CO})$ as measured by the single breath technique. Both were expressed as \% predicted. These data were available for 174 of the 185 subjects at presentation, 149 at 2 yrs and 136 at 4 yrs.

\begin{tabular}{|c|c|c|c|}
\hline \multirow[t]{2}{*}{ TABLE 1} & \multicolumn{3}{|c|}{$\begin{array}{l}\text { Chest radiograph staging of sarcoidosis patients } \\
\text { genotyped for the caspase recruitment domain } \\
\text { (CARD) } 15 \text {, and both CARD } 15 \text { and } C-C \\
\text { chemokine receptor }(C C R) 5 \text { single nucleotide } \\
\text { polymorphisms, at presentation, } 2 \text { yrs and } 4 \text { yrs }\end{array}$} \\
\hline & Presentation & $2 \mathrm{yrs}$ & $4 \mathrm{yrs}$ \\
\hline \multicolumn{4}{|l|}{ CARD15 } \\
\hline Subjects $n$ & 177 & 158 & 126 \\
\hline Stage 0 & $22(12.4)$ & 33 (20.9) & $37(29.4)$ \\
\hline Stage I & 33 (18.6) & $24(15.2)$ & $12(9.5)$ \\
\hline Stage II & $39(22.0)$ & $21(13.3)$ & $10(7.9)$ \\
\hline Stage III & $16(9.0)$ & $11(7.0)$ & $6(4.8)$ \\
\hline Stage IV & $67(37.9)$ & $69(43.7)$ & $61(48.4)$ \\
\hline \multicolumn{4}{|c|}{ CARD15 and CCR5 } \\
\hline Subjects $\mathrm{n}$ & 160 & 144 & 115 \\
\hline Stage 0 & $21(13.1)$ & $31(21.5)$ & $35(30.4)$ \\
\hline Stage I & $29(18.1)$ & $22(15.3)$ & $9(7.8)$ \\
\hline Stage II & 35 (21.9) & $17(11.8)$ & $10(8.7)$ \\
\hline Stage III & $14(8.8)$ & $11(7.6)$ & $6(5.2)$ \\
\hline Stage IV & $61(38.1)$ & $63(43.8)$ & $55(47.8)$ \\
\hline
\end{tabular}




\section{Data analysis}

Genotype and allele frequencies were determined by direct counting. Haplotypes were deduced by PHASE, version 2, a statistical haplotype reconstruction method [22].

Statistical analyses were performed using SPSS version 14 (SPSS, Chicago, IL, USA). Categorical data were analysed by Chi-squared contingency tables or Fisher's exact test as appropriate. Continuous data were analysed using the Mann-Whitney U-test or Kruskal-Wallis test as appropriate. A value of $\mathrm{p}<0.05$ was considered significant.

\section{RESULTS}

\section{CARD15 polymorphisms: unselected sarcoidosis cohort}

In order to determine whether there were any associations between sarcoidosis in general and CARD15 polymorphisms or haplotypes, allele and haplotype frequencies were compared for all sarcoidosis patients and the control population. These comparisons are shown in tables 2 and 3 . Table 2 summarises the allele frequencies of the five CARD15 polymorphisms investigated in the sarcoid and control populations. Both populations were in Hardy-Weinberg equilibrium for all genotype frequencies. No significant differences were found between the unselected sarcoidosis cohort and controls. Table 3 shows the CARD15 haplotypes and their frequencies: there were no significant differences in CARD15 haplotypes between the two groups.

Of the functional, Crohn's disease-associated polymorphisms, $2104 \mathrm{~T}$ occurred only on haplotype 4 , 3020insC on haplotype 5 and $2722 \mathrm{C}$ on haplotype $6.1761 \mathrm{G}$ occurred only on haplotype 1 , whereas 802 T occurred on haplotypes 3-6 (table 3).

\begin{tabular}{|c|c|c|c|}
\hline \multirow[t]{2}{*}{ Polymorphism } & \multirow{2}{*}{$\begin{array}{c}\text { Amino } \\
\text { acid }\end{array}$} & \multicolumn{2}{|c|}{ Allele frequency } \\
\hline & & Sarcoidosis ${ }^{\#}$ & Controls \\
\hline \multicolumn{4}{|l|}{802 (rs2066842) } \\
\hline C & Pro & 289 (78.1) & 505 (72.8) \\
\hline$T$ & Ser & $81(21.9)$ & $189(27.2)$ \\
\hline \multicolumn{4}{|c|}{1761 (rs1861759) } \\
\hline$T$ & Arg & 222 (60.0) & 439 (63.3) \\
\hline G & Arg & $148(40.0)$ & $255(36.7)$ \\
\hline \multicolumn{4}{|c|}{2104 (rs2066844) } \\
\hline C & Arg & 351 (94.9) & $658(94.8)$ \\
\hline$T$ & Trp & $19(5.1)$ & $36(5.2)$ \\
\hline \multicolumn{4}{|c|}{2722 (rs2066845) } \\
\hline G & Gly & 368 (99.5) & $684(98.6)$ \\
\hline C & Arg & $2(0.5)$ & $10(1.4)$ \\
\hline \multicolumn{4}{|c|}{3020 (rs2066847) } \\
\hline WT & & 367 (99.2) & $683(98.4)$ \\
\hline ins $C$ & Fs & $3(0.8)$ & $11(1.6)$ \\
\hline
\end{tabular}

\section{CARD15, CCR5 and radiographic stage IV disease in sarcoidosis}

Associations between CARD15 polymorphisms, the CCR5 $H H C$ haplotype and chest radiographic staging were investigated. Carriage of the CARD15 $2104 T$ polymorphism (haplotype 4) was associated with a higher frequency of radiographic stage IV disease than non-carriers at 4 yrs of follow up $(76.9 \%$ versus $45.1 \%$, OR $4.1,95 \%$ CI $1.0-15.5 ; \mathrm{p}=0.04)$. There were no significant differences between carriers and non-carriers of the other CARD15 polymorphisms or haplotypes and either parenchymal disease (stage II-IV) or stage IV disease at presentation, 2 yrs or 4 yrs.

We have previously shown an association between carriage of the CCR5 HHC haplotype and parenchymal disease at presentation (radiographic stage $\geqslant$ II versus stages 0 and I) [5]. In the current, larger, cohort (56 additional cases) the HHC haplotype was significantly increased in patients with stage IV compared with stage 0 -III disease at presentation $(\mathrm{n}=160,72.1 \%$ versus $46.5 \%$, OR 3.0, 95\% CI 1.5-5.9; $\mathrm{p}=0.003), 2$ yrs $(\mathrm{n}=144,68.3 \%$ versus $45.7 \%$, OR 2.6, 95\% CI 1.3-5.1; $\mathrm{p}=0.01)$ and 4 yrs $(\mathrm{n}=115$, $69.1 \%$ versus $46.7 \%, \mathrm{p}=0.02, \mathrm{OR}=2.6,95 \% \mathrm{CI}=1.2-5.5)$.

Having established the influence of the CCR5 HHC haplotype on radiographic stage IV disease and having seen for the first time a significant association between one of the functional Crohn's disease-associated CARD15 SNPs (2104T, haplotype 4) and stage IV disease, we investigated the influence of $2104 \mathrm{~T}$ in sarcoidosis patients subtyped according to their CCR5 HHC haplotype status. Patients were divided into four groups: 1) Group $1(n=7)$ : carriage of both CCR5 HHC and CARD15 $2104 T$; 2) Group $2(\mathrm{n}=83)$ : carriage of only CCR5 HHC; 3) Group $3(\mathrm{n}=8)$ : carriage of only CARD15 2104T; and 4) Group $4(n=62)$ : carriage of neither.

All seven of the subjects carrying the HHC haplotype and CARD15 $2104 T$ (haplotype 4) had radiographic stage IV disease at presentation, 2 yrs and 4 yrs (fig. 1).

No differences were found when the subgroups were similarly analysed for change in chest radiograph status (at 4 yrs followup versus at presentation).

\begin{tabular}{|c|c|c|c|c|c|c|c|}
\hline \multirow[t]{3}{*}{ TABLE 3} & \multicolumn{7}{|c|}{$\begin{array}{l}\text { Caspase recruitment domain }(C A R D) 15 \\
\text { haplotypes and their carriage frequencies in } \\
\text { sarcoidosis and controls }\end{array}$} \\
\hline & \multirow[t]{2}{*}{802} & \multirow[t]{2}{*}{1761} & \multirow[t]{2}{*}{2104} & \multirow[t]{2}{*}{2722} & \multirow[t]{2}{*}{3020} & \multicolumn{2}{|c|}{ Carriage frequency } \\
\hline & & & & & & Sarcoidosis ${ }^{\#}$ & Controls \\
\hline Haplotype 1 & C & G & $\mathrm{C}$ & $G$ & WT & $123(66.5)$ & $212(61.1)$ \\
\hline Haplotype 2 & C & T & $\mathrm{C}$ & $G$ & WT & $116(62.7)$ & $205(59.1)$ \\
\hline Haplotype 3 & $\mathbf{T}$ & $\mathrm{T}$ & C & $G$ & WT & $52(28.1)$ & $122(35.2)$ \\
\hline Haplotype 4 & $\mathbf{T}$ & $\mathrm{T}$ & $\mathbf{T}$ & $G$ & WT & $17(9.2)$ & $35(10.1)$ \\
\hline Haplotype 5 & $\mathbf{T}$ & $\mathrm{T}$ & $\mathrm{C}$ & $G$ & ins C & $3(1.6)$ & $11(3.2)$ \\
\hline Haplotype 6 & $\mathbf{T}$ & T & C & C & WT & $2(1.1)$ & $10(2.9)$ \\
\hline
\end{tabular}

Data are presented as $n$ (\%). Bold signifies a variant allele. WT: wild-type; ins C insertion C. ${ }^{\#}: n=185 ; ": n=347$. 


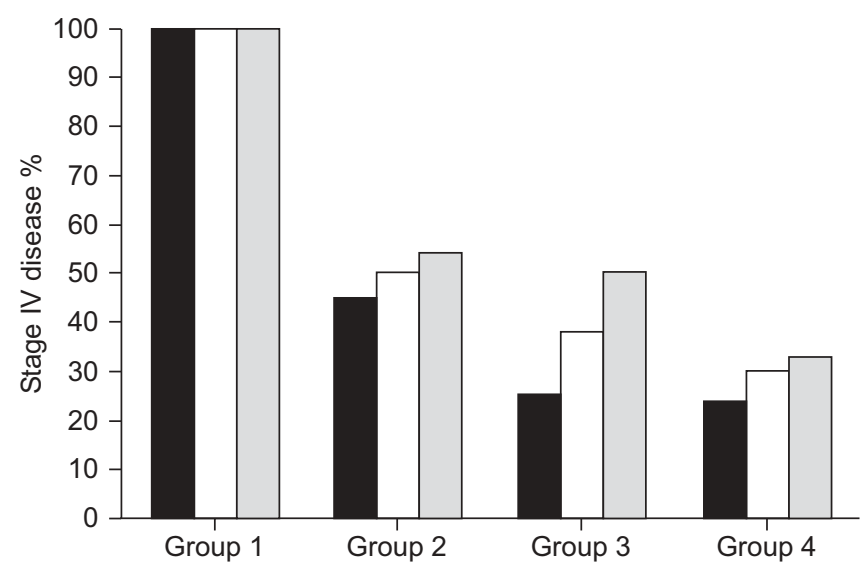

FIGURE 1. Frequencies of chest radiograph stage IV disease related to $C-C$ chemokine receptor (CCR)5 HHC and caspase recruitment domain (CARD15) $2104 T$ (haplotype 4) status. Group 1 ( $n=7$ at presentation and 2 yrs and $n=6$ at 4 yrs): carriage of both CCR5 HHC and CARD15 2104T; Group 2 ( $\mathrm{n}=83$ at presentation, $n=73$ at 2 yrs and $n=60$ at $4 \mathrm{yrs}$ ): carriage of only CCR5 HHC; Group 3 ( $\mathrm{n}=8$ at presentation and $2 \mathrm{yrs}$ and $\mathrm{n}=6$ at $4 \mathrm{yrs}$ ): carriage of only CARD15 2104T; Group 4 ( $n=62$ at presentation, $n=56$ at $2 \mathrm{yrs}$ and $\mathrm{n}=43$ at $4 \mathrm{yrs}$ ): carriage of neither. All of the subjects carrying CCR5 HHC and CARD15 $2104 T$ had stage IV disease at presentation ( $p=0.0003$, Kruskal-Wallis test), and at $2 \mathrm{yrs}$ and $4 \mathrm{yrs}$.

口: at presentation; $\square: 2 \mathrm{yrs} ; \quad 10: 4 \mathrm{yrs}$.

The CARD15 $802 T$ polymorphism in combination with the HHC haplotype was also significantly associated with radiographic stage IV disease $(\mathrm{p}=0.002$ at presentation, 2 yrs and 4 yrs) (fig. 2; groups as described for fig. 1 but for $802 T$ carriage rather than 2104T). Further analysis revealed, however, that this was a consequence of the known linkage disequilibrium [11] between $802 T$ and $2104 T$ (table 3).

No significant associations were found for $2722 \mathrm{C}$, 3020ins C or $1761 \mathrm{G}$, or their respective haplotypes.

\section{CARD15 polymorphisms and pulmonary function tests}

To clarify whether the carriage of CARD15 polymorphisms and/or haplotypes acted as an index for disease severity, the possession of CARD15 polymorphisms (and hence haplotypes, table 3) was investigated in relation to lung function tests.

Table 4 shows the carriage of the $802 \mathrm{~T}, 1761 \mathrm{G}$ (haplotype 1) and $2104 \mathrm{~T}$ (haplotype 4) alleles and lung function tests at presentation, 2 yrs and 4 yrs. Carriage of the CARD15 1761G allele (haplotype 1) was associated with better lung function, as defined by $T \mathrm{~L}, \mathrm{CO}$, than non-carriage of the $G$ allele at presentation $(p=0.001), 2$ yrs $(p=0.006)$ and 4 yrs $(p=0.002)$. The differences were significant at all time points, and interestingly a clear gene-dose effect can be seen at presentation: GG homozygotes have better TL,CO than TG heterozygotes, whereas TT homozygotes have the worst TL,CO (fig. 3).

Figure 4 shows the mean comparisons of TL,CO with CARD15 1761 genotypes at presentation, 2 yrs and 4 yrs. Carriers of the $1761 \mathrm{G}$ allele had a higher FEV1 $(\mathrm{p}=0.02)$ at presentation compared with non-carriers. These patients also had a higher FVC at presentation $(p=0.01)$; there was a trend towards significance for FVC at 2 yrs $(\mathrm{p}=0.07)$; and at 4 yrs the difference

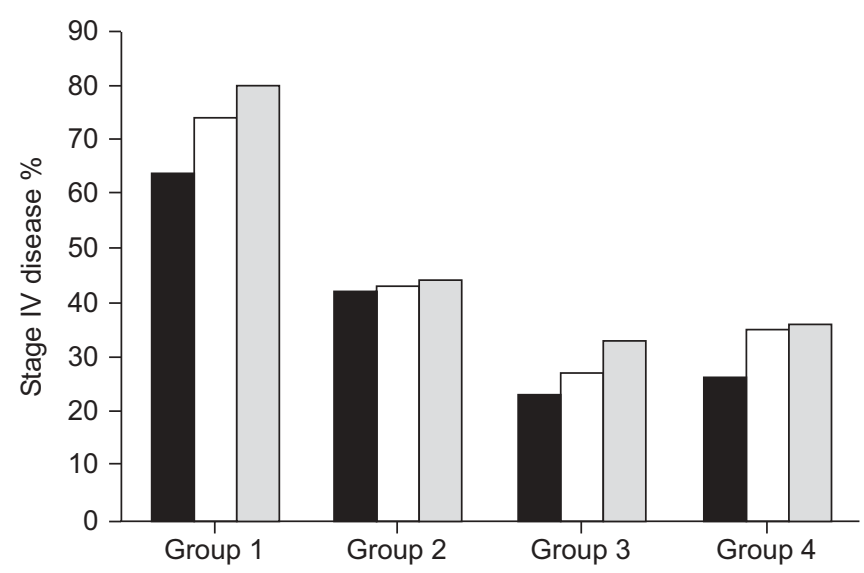

FIGURE 2. Frequencies of chest radiograph stage IV disease related to $C$-C chemokine receptor (CCR)5 HHC haplotype and caspase recruitment domain (CARD)15 802T (haplotypes 3-6) status. Group 1 ( $n=28$ at presentation, $n=27$ at 2 yrs and $n=25$ at 4 yrs): carriage of both CCR5 HHC and CARD15 802T; Group 2 $(\mathrm{n}=62$ at presentation, $\mathrm{n}=53$ at $2 \mathrm{yrs}$ and $\mathrm{n}=41$ at $4 \mathrm{yrs}$ ): carriage of only CCR5 HHC; Group 3 ( $n=31$ at presentation, $n=30$ at 2 yrs and $n=24$ at 4 yrs): carriage of only CARD15 802T; Group $4(n=39$ at presentation, $n=34$ at 2 yrs and $n=25$ at 4 yrs): carriage of neither. The CARD15 $802 T$ polymorphism in combination with the CCR5 HHC haplotype was also significantly associated with stage IV disease at presentation $(p<0.005), 2$ yrs $(p<0.005)$, and 4 yrs $(p<0.005)$, Kruskal-Wallis test.

口: at presentation; $\square: 2 \mathrm{yrs} ; \quad \square: 4$ yrs.

was again significant $(\mathrm{p}=0.02)$ (table 4$)$. Of note, this cohort with better lung function did not possess haplotype 4 , which includes the $2104 \mathrm{~T}$ allele.

Consistent with the worse radiographic stage associations, patients carrying the $2104 \mathrm{~T}$ allele (haplotype 4) had a worse median FEV1 at presentation $(79.3 \%$ versus $92.3 \%$; $\mathrm{p}=0.04)$, 2 yrs $(73.8 \%$ versus $93.3 \% ; \mathrm{p}=0.02)$ and $4 \mathrm{yrs}(77.6 \%$ versus $92.4 \% ; p=0.05)$, and a worse median FVC at 4 yrs follow-up $(89.3 \%$ versus $100.3 \% ; p=0.04)$ than patients not carrying the 2104T allele.

No significant differences were seen between the carriage of $802 T$, 3020ins C or 2722C, and pulmonary function tests.

\section{DISCUSSION}

Sarcoidosis and Crohn's disease are both characterised by inflammation, granuloma formation and their systemic nature, with frequent extrapulmonary and extraintestinal manifestations respectively. They both display considerable phenotypic heterogeneity and degrees of disease severity, and important genetic associations have been identified for both conditions. The similarities between sarcoidosis and Crohn's disease have prompted several studies of the Crohns susceptibility gene CARD15/NOD2 in unselected sarcoidosis cohorts [15-18]. Of these, one has reported an association with the 2722C (G908R) variant [18] and one [17] found an increased transmission of two of the alleles but concluded that these mutations played no major role in their study population.

The CARD15 polymorphisms studied are specifically associated with Crohn's disease subtypes, and it was hypothesised that they may be associated with subtypes of sarcoidosis. The CCR5 gene has also been associated with both diseases [5, 6], 


\begin{tabular}{|c|c|c|c|c|c|c|c|c|c|c|c|c|}
\hline \multirow[t]{3}{*}{ TABLE 4} & \multicolumn{12}{|c|}{$\begin{array}{l}\text { Carriage of caspase recruitment domain (CARD) } 15 \text { 802T, } 1761 \mathrm{G} \text { (haplotype 1) and } 2104 \mathrm{~T} \text { (haplotype 4) alleles in } \\
\text { relation to lung function tests at presentation, } 2 \text { yrs and } 4 \text { yrs }\end{array}$} \\
\hline & & \multicolumn{3}{|c|}{$802 T$} & \multicolumn{4}{|c|}{$1761 G$ (haplotype 1) } & \multicolumn{4}{|c|}{$2104 T$ (haplotype 4) } \\
\hline & $\mathbf{n}$ & FEV $_{1}$ & FVC & $T L, C O$ & $\mathbf{n}$ & FEV $_{1}$ & FVC & $T L, C O$ & $\mathbf{n}$ & FEV $_{1}$ & FVC & $T \mathrm{~L}, \mathrm{CO}$ \\
\hline Presentation & 174 & & & & & & & & & & & \\
\hline $\mathrm{p}$-value & & 0.42 & 0.5 & 0.2 & & 0.02 & 0.01 & 0.001 & & 0.04 & 0.3 & 0.07 \\
\hline 2 yrs & 148 & & & & & & & & & & & \\
\hline Carrier & 59 & 94.3 & 101.1 & 77.45 & 99 & 92.3 & 101.3 & 80.8 & 15 & 73.8 & 94.1 & 71.9 \\
\hline Non-carrier & 89 & 91.2 & 98.7 & 78.6 & 50 & 89.7 & 97.9 & 71.9 & 133 & 93.3 & 99.9 & 79.5 \\
\hline$p$-value & & 0.6 & 0.9 & 0.5 & & 0.2 & 0.07 & 0.006 & & 0.02 & 0.2 & 0.1 \\
\hline
\end{tabular}

Data are presented as median \% predicted values, unless otherwise stated. Bold signifies a significant value. FEV1: forced expiratory volume in $1 \mathrm{~s}$; FVC: forced vital capacity; $T L, C O$ : transfer factor of the lung for carbon monoxide.

and this gene was further investigated to allow subclassification of the cohort on the basis of their CCR5 haplotype.

Significant associations have been demonstrated between both a functional CARD15 SNP (2104T, haplotype 4) and a synonymous CARD15 SNP (1761G, haplotype 1), and severe pulmonary sarcoidosis phenotypes. An association between the CCR5 HHC haplotype and chest radiographic stage IV disease has been established. Of note, all patients possessing both the CCR5 HHC haplotype and CARD15 $2104 T$ allele had radiographic stage IV disease at presentation.

Patients possessing the functional $2104 \mathrm{~T}$ allele (haplotype 4) had worse FEV1 values at presentation, 2 yrs and 4 yrs, as well as worse FVC at 4 yrs. The carriage of this polymorphism was

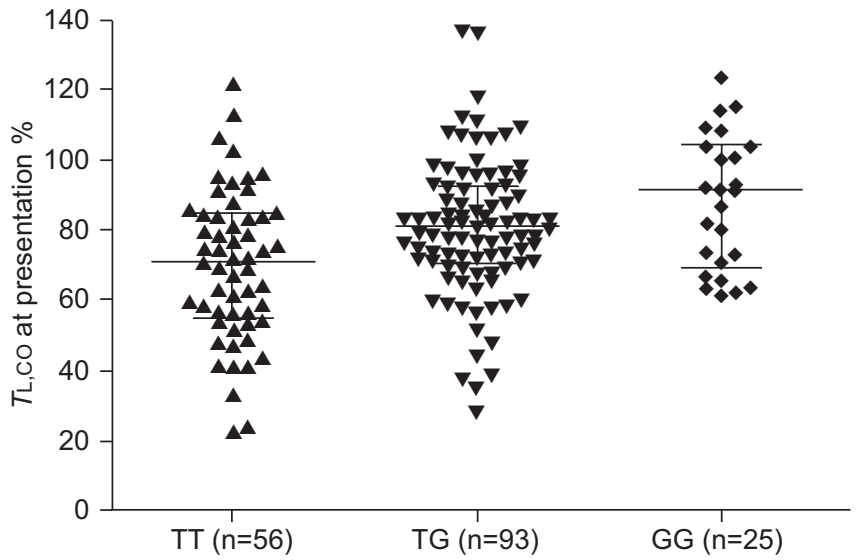

FIGURE 3. Influence of caspase recruitment domain (CARD)15 1761 genotype on the transfer factor of the lung for carbon monoxide $(T L, C O)$. There was a significant gene-dose effect between CARD15 1761 genotype (GG>TG>TT) and the level of $T L, C O$ at presentation $(p=0.001)$. Median and interquartile ranges are shown by horizontal lines. also associated with an increased risk of radiographic stage IV disease at 4 yrs.

The CARD15 gene product plays an important role in innate immunity by influencing recognition of specific bacterial patterns, and this is impaired in those possessing the Crohn's disease-associated functional variants. The precise mechanism through which these variants result in increased disease susceptibility remains uncertain. Transient transfection experiments have suggested that the impaired binding of muramyl

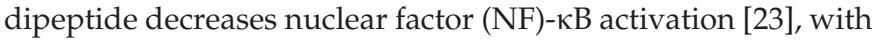
consequent impaired microbial clearance. In CARD15 mutant mice, however, increased downstream NF- $\mathrm{B}$ signalling has been demonstrated [24] with subsequent inflammation. The association of one of the functional mutations with a severe

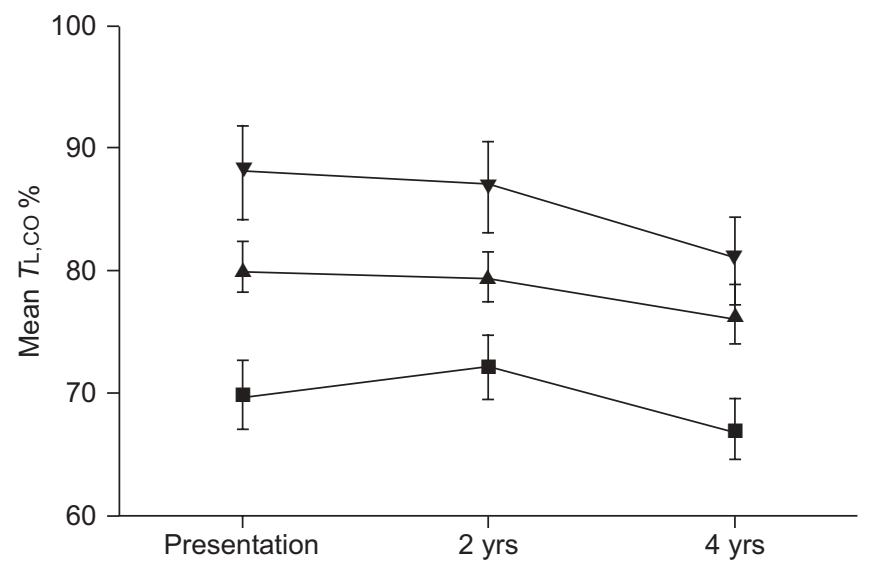

FIGURE 4. Mean \pm SEM comparisons of transfer capacity of the lung for carbon monoxide $(T L, C O)$ with caspase recruitment domain (CARD)15 1761 genotypes at presentation, 2 yrs and 4 yrs. Individuals with the $G G$ genotype $(>T G>T T$ ) had significantly higher mean $T L, C O$ levels at presentation $(p=0.001), 2$ yrs $(p=0.009)$ and 4 yrs $(p=0.003)$. $\mathbf{\square}: T$; $\mathbf{\Lambda}:$ TG; $\mathbf{\nabla}: G G$ 
pulmonary sarcoidosis phenotype offers an intriguing insight into possible dysregulated responses to bacteria in this cohort.

We have previously found associations between the CCR5 HHC haplotype and parenchymal lung disease in a cohort of 104 British patients [5]. In this larger cohort, we have elucidated a significant association with pulmonary radiographic stage IV disease. These findings are consistent with our previous results, suggesting that dysfunction of this gene, of major importance in T-cell activation, could result in abnormal trafficking of T-cells to the lung and hence parenchymal abnormalities culminating in fibrosis [5].

All patients carrying the CCR5 HHC haplotype and the CARD15 $2104 T$ polymorphism had pulmonary stage IV disease at presentation. It is of interest that these two genes are both involved in innate immunity as discussed above. CARD15-deficient mice have been shown to lack the normal inhibition of the toll-like receptor (TLR) 2-mediated NF- $\kappa B$ response, with consequent T-helper (Th) cell type 1 overactivation [25]. CCR5 also enhances T-cell activation [26]. It is hypothesised that the possession of CARD15 $2104 T$ contributes to an impaired response to bacteria, whether commensal or pathogenic, with subsequent abnormal T-cell activation that is further enhanced by the possession of CCR5 HHC in this cohort of patients.

The CARD15 $1761 G$ allele, present in $40 \%$ of the sarcoidosis cohort, was associated with significantly better lung function parameters (not only TL,CO but also FVC) than the wild-type allele at all time-points. This suggests that carriage of this polymorphism is associated with less diffuse lung disease. A significant gene-dose effect was also observed (fig. 3). In Crohn's disease, this polymorphism is protective: there is a lower frequency of Crohn's disease in those possessing the CARD15 1761G allele [11]. This suggests that the polymorphism confers a favourable phenotype. It is, however, a synonymous SNP.

Possible explanations for the association of this synonymous SNP with a specific disease phenotype may include linkage disequilibrium with another, functional SNP, as yet unidentified. This hypothesis would suggest that it is acting as a marker. Alternatively, there has been considerable interest in "silent" polymorphisms, and a functional study has shown that the product of a synonymous polymorphism can differ significantly from the wild-type protein [27]. It was hypothesised that the synonymous polymorphism may affect the timing of cotranslational folding and so alter the structure of binding sites. Further functional studies of the 1761G SNP will be required to investigate this.

In this study, chest radiographs were evaluated independently by two experienced pulmonary radiologists and chest radiographic appearances remain the accepted means of staging sarcoidosis [19]. However, pulmonary computed tomography (CT) scoring may be a more sensitive means of assessing lung disease pattern, including fibrosis and disease severity [28], and further studies investigating carriage of these genetic polymorphisms in relation to CT score may serve to confirm the association between lung fibrosis and these genes. Indeed, the relative insensitivity of chest radiographs may explain the lack of association found for CARD15 1761G and radiographic stage, when significant associations with lung function were found. Quantification of lung function change with continuous variables may be more precise than the categorical, descriptive chest radiographic staging system, and thus more likely to be able to identify associations [29].

A recent study using blood mononuclear cells has shown that a combination of NOD2 and TLR-2 ligand stimulation induced a higher secretion of the pro-inflammatory cytokines tumour necrosis factor (TNF)- $\alpha$ and interleukin (IL)- $1 \beta$ in sarcoidosis patients than healthy controls [30]. These cytokines are important in determining the inflammatory response in active sarcoidosis, and indeed granuloma formation. It will be informative to perform further such studies in individuals subclassified according to their CARD15 genotype. Specifically, it would be of interest to investigate whether those possessing the $1761 \mathrm{G}$ allele have a reduced predisposition to TNF- $\alpha$ and IL$1 \beta$ secretion compared with those with the wild-type allele, and whether those with the $2104 T$ polymorphism have significantly greater secretion of these cytokines.

In conclusion, neither the CARD15 nor the CCR5 variants were more prevalent overall in the sarcoidosis cohort than the controls, confirming the hypothesis that these are not disease susceptibility genes but rather are associated with disease modification and/or progression after sarcoidosis is established. These results provide further evidence for genetic heterogeneity in determining the phenotype in sarcoidosis.

\section{SUPPORT STATEMENT}

Financial support was provided by the Asmarley Trust and the Broad Medical Research Program.

\section{STATEMENT OF INTEREST}

None declared.

\section{ACKNOWLEDGEMENTS}

The authors would like to thank the Asmarley Trust and the Broad Medical Research Program for their financial support and F. Woodhead for helpful discussions.

\section{REFERENCES}

1 Sato H, Grutters JC, Pantelidis P, et al. HLA-DQB1*0201: a marker for good prognosis in British and Dutch patients with sarcoidosis. Am J Respir Cell Mol Biol 2002; 27: 406-412.

2 Voorter CE, Drent M, van den Berg-Loonen EM. Severe pulmonary sarcoidosis is strongly associated with the haplotype HLA-DQB1*0602-DRB1*150101. Hum Immunol 2005; 66: 826-835.

3 Ahmad T, Armuzzi A, Bunce M, et al. The molecular classification of the clinical manifestations of Crohn's disease. Gastroenterology 2002; 122: 854-866

4 Orchard TR, Chua CN, Ahmad T, et al. Uveitis and erythema nodosum in inflammatory bowel disease: clinical features and the role of HLA genes. Gastroenterology 2002; 123: 714-718.

5 Spagnolo P, Renzoni EA, Wells AU, et al. C-C chemokine receptor 5 gene variants in relation to lung disease in sarcoidosis. Am J Respir Crit Care Med 2005; 172: 721-728.

6 Rector A, Vermeire S, Thoelen I, et al. Analysis of the CC chemokine receptor 5 (CCR5) delta-32 polymorphism in inflammatory bowel disease. Hum Genet 2001; 108: 190-193.

7 Hugot JP, Chamaillard M, Zouali $\mathrm{H}$, et al. Association of NOD2 leucine-rich repeat variants with susceptibility to Crohn's disease. Nature 2001; 411: 599-603. 
8 Ogura Y, Bonen DK, Inohara N, et al. A frameshift mutation in NOD2 associated with susceptibility to Crohn's disease. Nature 2001; 411: 603-606.

9 Ogura Y, Inohara N, Benito A, et al. Nod2, a Nod1/Apaf-1 family member that is restricted to monocytes and activates NF-kappaB. J Biol Chem 2001; 276: 4812-4818.

10 Ahmad T, Marshall S, Jewell D. Genotype-based phenotyping heralds a new taxonomy for inflammatory bowel disease. Curr Opin Gastroenterol 2003; 19: 327-335.

11 Lesage S, Zouali H, Cezard JP, et al. CARD15/NOD2 mutational analysis and genotype-phenotype correlation in 612 patients with inflammatory bowel disease. Am J Hum Genet 2002; 70: 845-857.

12 Arostegui JI, Arnal C, Merino R, et al. NOD2 gene-associated pediatric granulomatous arthritis: Clinical diversity, novel and recurrent mutations, and evidence of clinical improvement with interleukin-1 blockade in a Spanish cohort. Arthritis Rheum 2007; 56: 3805-3813.

13 Miceli-Richard C, Lesage S, Rybojad M, et al. CARD15 mutations in Blau syndrome. Nat Genet 2001; 29: 19-20.

14 Kanazawa N, Okafuji I, Kambe N, et al. Early-onset sarcoidosis and CARD15 mutations with constitutive nuclear factor-kappaB activation: common genetic etiology with Blau syndrome. Blood 2005; 105: 1195-1197.

15 Ho LP, Merlin F, Gaber K, et al. CARD 15 gene mutations in sarcoidosis. Thorax 2005; 60: 354-355.

16 Milman N, Nielsen OH, Hviid TV, et al. CARD15 single nucleotide polymorphisms 8, 12 and 13 are not increased in ethnic Danes with sarcoidosis. Respiration 2007; 74: 76-79.

17 Schurmann M, Valentonyte R, Hampe J, et al. CARD15 gene mutations in sarcoidosis. Eur Respir J 2003; 22: 748-754.

18 Gazouli M, Koundourakis A, Ikonomopoulos J, et al. CARD15/ NOD2, CD14, and toll-like receptor 4 gene polymorphisms in Greek patients with sarcoidosis. Sarcoidosis Vasc Diffuse Lung Dis 2006; 23: 23-29.

19 Statement on sarcoidosis. Joint Statement of the American Thoracic Society (ATS), the European Respiratory Society (ERS) and the World Association of Sarcoidosis and Other
Granulomatous Disorders (WASOG) adopted by the ATS Board of Directors and by the ERS Executive Committee, February 1999. Am J Respir Crit Care Med 1999; 160: 736-755.

20 Bunce M, O'Neill CM, Barnardo MC, et al. Phototyping: comprehensive DNA typing for HLA-A, B, C, DRB1, DRB3, DRB4, DRB5 \& DQB1 by PCR with 144 primer mixes utilizing sequence-specific primers (PCR-SSP). Tissue Antigens 1995; 46: 355-367.

21 Welsh K, Bunce M. Molecular typing for the MHC with PCR-SSP. Rev Immunogenet 1999; 1: 157-176.

22 Stephens M, Smith NJ, Donnelly P. A new statistical method for haplotype reconstruction from population data. Am J Hum Genet 2001; 68: 978-989.

23 Inohara N, Ogura Y, Fontalba A, et al. Host recognition of bacterial muramyl dipeptide mediated through NOD2. Implications for Crohn's disease. J Biol Chem 2003; 278: 5509-5512.

24 Maeda S, Hsu LC, Liu H, et al. Nod2 mutation in Crohn's disease potentiates NF-kappaB activity and IL-1beta processing. Science 2005; 307: 734-738

25 Watanabe T, Kitani A, Murray PJ, et al. NOD2 is a negative regulator of Toll-like receptor 2-mediated $\mathrm{T}$ helper type 1 responses. Nat Immunol 2004; 5: 800-808.

26 Molon B, Gri G, Bettella M, et al. T cell costimulation by chemokine receptors. Nat Immunol 2005; 6: 465-471.

27 Kimchi-Sarfaty C, Oh JM, Kim IW, et al. A "silent" polymorphism in the MDR1 gene changes substrate specificity. Science 2007; 315: 525-528.

28 Hansell DM, Milne DG, Wilsher ML, et al. Pulmonary sarcoidosis: morphologic associations of airflow obstruction at thin-section CT. Radiology 1998; 209: 697-704.

29 Keogh BA, Crystal RG. Pulmonary function testing in interstitial pulmonary disease. What does it tell us? Chest 1980; 78: 856-964.

30 Wiken M, Grunewald J, Eklund A, et al. Higher monocyte expression of TLR2 and TLR4, and enhanced pro-inflammatory synergy of TLR2 with NOD2 stimulation in sarcoidosis. J Clin Immunol 2009; 29: 78-89. 